\title{
Identificação de localizações estratégicas de unidades de armazenamento, beneficiamento e distribuição para extrativismo do pequi
}

\section{Kamilla Nunes Froes ${ }^{1}$, Dario Alves de Oliveira ${ }^{1}$, Marcos Esdras Leite $^{2}$ e Narciso Ferreira dos Santos Neto ${ }^{3}$}

\author{
${ }^{1}$ Universidade Estadual de Montes Claros. Programa de Pós-Graduação em \\ Biotecnologia. Montes Claros-MG, Brasil (CEP 39401-089). \\ ${ }^{2}$ Universidade Estadual de Montes Claros. Programa de Pós-Graduação em \\ Geografia. Montes Claros-MG, Brasil (CEP 39401-089). \\ ${ }^{3}$ Universidade Estadual de Montes Claros. Programa de Pós-Graduação em \\ Modelagem Computacional e Sistemas. Montes Claros-MG, Brasil (CEP 39401-089).
}

Resumo. A atividade extrativista do pequi é de grande relevância para a mesorregião Norte do Estado de Minas Gerais. Desta forma, o presente trabalho objetivou identificar localizações estratégicas de unidades de armazenamento, beneficiamento e distribuição para extrativismo do pequi na referida região, a fim de auxiliar seu fortalecimento. Para tanto, foi utilizado um modelamento matemático de localização de instalações (Location-Allocation) do tipo p-medianas não capacitado através de Sistemas de Informações Geográficas, o que permitiu a execução de um método heurístico de localização-alocação. Para isso, foram inseridas ao software ArcGIS 10.1 a localização de 118 comunidades extrativistas de pequi em 22 municípios do norte do estado de Minas Gerais, 26.407,32 km de feições vetorizadas do sistema viário das regiões contempladas e 3.711 pontos potenciais para a instalação das unidades extrativistas. As simulações executadas demonstram a possibilidade de implantação de cinco unidades extrativistas que permitem minimizar o tempo gasto de deslocamento entre os pontos de demanda (comunidades) e as facilidades (unidades extrativistas) e com localizações previstas para os Municípios de Lontra, Mirabela, Januária, Japonvar, Chapada Gaúcha e Brasília de Minas. Constatou-se que com o aumento do número de unidades de armazenamento, beneficiamento e distribuição para extrativismo do pequi, diminui-se o tempo total gasto de deslocamento em relação às comunidades extrativistas, o que consequentemente reduzirá os custos com transporte, sendo, portanto, a implantação de cinco unidades extrativistas a simulação mais vantajosa.

Palavras-chave: Extrativismo; Modelamento matemático; Recursos florestais não madeireiros; Sistemas de Informações Geográficas.

Recebido

$29 / 10 / 2019$

Aceito

$27 / 12 / 2019$

Publicado

$31 / 12 / 2019$

Acesso aberto

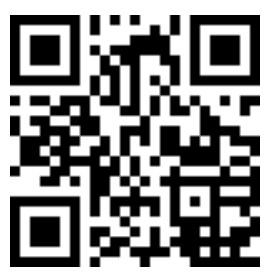

ORCID

(D) 0000-0003-2215-2593 Kamilla Nunes Froes

(D) $0000-0002-8161-4607$ Dario Alves de Oliveira

(D) 0000-0002-9020-6445 Marcos Esdras Leite

(1) 0000-0002-1742-3515

Narciso Ferreira dos

Santos Neto 


\begin{abstract}
Identification of strategic locations of storage units, processing and distribution for pequi extractivism. The extractive activity of pequi is of great relevance to the northern mesoregion of the State of Minas Gerais, Brazil. In this way, the present work aimed to identify strategic locations of storage, processing and distribution units for pequi extraction in the region, in order to assist fortification. In order to do so, a mathematical model of Location-Allocation of type uncapacitated p-median used through Geographic Information Systems, which allowed the execution of a localization-allocation heuristic method. In order to do so, we inserted into the ArcGIS 10.1 software the location of 118 extractive communities of pequi in 22 municipalities in the north of the State of Minas Gerais, $26,407.32 \mathrm{~km}$ of vectorial features of the road system of the contemplated regions and 3,711 potential points for the installation of the extractive units. The simulations carried out for the implantation of 5 extractivist units sought to minimize the time spent displacing between the demand points (communities) and the facilities (extractive units) and contemplated the Municipalities of Lontra, Mirabela, Januária, Japonvar, Chapada Gaúcha and Brasília de Minas. It was verified that with the increase in the number of storage, processing and distribution units for pequi extractivism, the total time of displacement in relation to the extractivist communities is reduced, which consequently reduces transportation costs, the implementation of five extractive units the most advantageous simulation.
\end{abstract}

Keywords: Extractivism; Mathematical modeling; Non-timber forest resources; Geographic Information Systems.

\section{Introdução}

0 pequizeiro Caryocar brasiliense Camb. é uma espécie de planta arbórea e pertencente à Família Caryocaraceae. (Rodrigues et al., 2015), considerada endêmica no Cerrado (Magalhães Filho et al., 2017) e uma das mais conhecidas do bioma no Brasil, pelo conjunto de valores que representa para populações tradicionais quando são considerados aspectos gastronômicos, econômicos, culturais, ecológicos e medicinais (Silva e Tubaldini, 2013).

0 pequi é o principal produto florestal não madeireiro do Cerrado (Afonso e Ângelo, 2009), e sua exploração realizada pelas populações tradicionais é considerada uma prática ambientalmente sustentável e economicamente rentável, uma vez que, garante a preservação da vegetação nativa e, consequentemente, explorações futuras (Pedrozo et al., 2011).

Os produtos com utilização do fruto, a serem considerados, podem ser divididos em três segmentos: pequi em caroço, pequi despolpado sem caroço e a castanha extraída da semente. A cadeia extrativista do fruto pode ser dividida em três macrossegmentos: obtenção da matéria-prima, industrialização e comercialização (Candido et al., 2012).

Dentre as mesorregiões brasileiras, o norte do estado de Minas Gerais é caracterizado pelo alto potencial de produção e exploração do pequi (Silva e Tubaldini, 2013), sendo esta a área escolhida para o desenvolvimento do presente trabalho, onde, embora ainda apresente a cadeia produtiva do fruto sobre condições de informalidade, tem 
contribuído para o desenvolvimento de diversos municípios da região (Santos et al., 2017).

Com base no exposto, o presente trabalho propõe um modelo de localizações estratégicas de unidades de armazenamento, beneficiamento e distribuição para extrativismo do pequi na mesorregião norte do estado de Minas Gerais a fim de auxiliar no fortalecimento de sua cadeia produtiva.

\section{Material e métodos}

A fim de identificar os municípios norte mineiros com maior potencial de produção e exploração extrativista do pequi na região norte do estado de Minas Gerais e promover o direcionamento eficiente de unidades de armazenamento, beneficiamento e distribuição para extrativismo do pequi, foram selecionados como amostra, os municípios com produção anual superior a 200 toneladas em 2014 (Tabela 1), a partir do banco de dados do Instituto Brasileiro de Geografia e Estatística (IBGE), conforme proposição também realizada por Santos et al. (2017).

Tabela 1. Quantidade produzida de pequi nos principais municípios produtores da mesorregião norte do estado de Minas Gerais no ano de 2014.

\begin{tabular}{lc}
\hline Municípios & $\begin{array}{c}\text { Quantidade produzida no ano de 2014 em } \\
\text { toneladas }\end{array}$ \\
\hline Japonvar & 993 \\
\hline Urucuia & 948 \\
\hline Ubaí & 924 \\
\hline São Francisco & 899 \\
\hline Mirabela & 861 \\
\hline Lontra & 850 \\
\hline Campo Azul & 800 \\
\hline Chapada Gaúcha & 757 \\
\hline Patis & 713 \\
\hline Ponto Chique & 696 \\
\hline Brasília de Minas & 616 \\
\hline Ibiracatu & 585 \\
\hline São João da Ponte & 523 \\
\hline Luislândia & 520 \\
\hline Montes Claros & 500 \\
\hline Varzelândia & 431 \\
\hline Pintópolis & 418 \\
\hline Icaraí de Minas & 253 \\
\hline
\end{tabular}

Fonte: IBGE (2019).

Foram selecionados também os Municípios de Francisco Dumont, Jequitaí; Coração de Jesus, Buritizeiro, Lagoa dos Patos, Januária, Bocaiuva e Guaraciama, que, apesar de não demonstrarem produção expressiva nos dados observados no IBGE, são popularmente caraterizados pela exploração do fruto (Santos et al., 2017).

Para identificação das localizações ideais das unidades extrativistas para armazenamento, beneficiamento e distribuição do fruto foi proposto a aplicação de um modelamento matemático de localização de instalações 
(Location-Allocation) para otimização da cadeia extrativista do pequi. Os Problemas de Localização-Alocação mostram-se como importantes linhas de pesquisas (Camara et al., 2016) por serem frequentemente utilizados para determinar os lugares ideais para localização de unidades para desenvolvimento das atividades consideradas principais e estratégicas (Costa et al., 2013). Crainic e Laporte (1997) classificam os modelos em LocalizaçãoAlocação como sendo de Cobertura, $p$ Centro e $p$-Medianas.

0 modelo matemático de localização de instalações escolhido para identificação das localizações ideais das unidades de armazenamento, beneficiamento e distribuição do pequi, por melhor se adequar aos objetivos pretendidos, foi o modelo p-medianas (Minimize Impedance/p-median) não capacitado, executado com o auxílio de Sistemas de Informações Geográficas (SIG), no módulo Location-Allocation disponível na extensão Network Analyst do software ArcGIS 10.1, o que permitiu a execução de um método heurístico de localização-alocação.

Em modelos do tipo p-medianas, um número pré-determinado de facilidades é localizado de forma a minimizar a distância média, tempo ou custo de deslocamento de uma facilidade em relação às demandas (Calache e Camargo, 2016). Segundo Brondani, (2013), a formulação dos problemas pmedianas como um modelo de programação linear inteira $0-1$ pode ser expressa por:

$$
V p=\min \sum_{i=1}^{n} \sum_{j=1}^{n} d_{i j} X_{i j}
$$

Sujeito a:

$$
\begin{aligned}
& \sum_{i=1}^{n} X_{i j}=1 ; j \in N . \\
& \sum_{i=1}^{n} X_{i j}=p \\
& X_{i j} \leq 0 X_{i i} ; i, j \in \ldots \ldots . . . \\
& X_{i j} \in(0,1) ; i, j \in N
\end{aligned}
$$

\section{Em que:}

$\left(d_{i j}\right)_{n \times n}$ é uma matriz simétrica de tempo com $d_{i j}=0, \forall i$ caso contrário;

$\left(x_{i j}\right)_{n x n}$ é a matriz de alocação, $\operatorname{com} x_{i j}=1$ se o vértice $i$ é alocado ao vértice $j$, e $x i j=0$,

$x_{i i}=1$ se o vértice i for uma mediana, e $x_{i i}=0$ em caso contrário;

p é o número de unidades de armazenamento, beneficiamento e distribuição do pequi a serem localizadas;

n é o número de vértices e $N=\{1, \ldots, n\}$.

O modelo de localização do tipo p-medianas é reconhecido como um problema NP-difícil (Garey e Jonhson, 1979), cujo esforço computacional para a solução cresce exponencialmente. Por sua vez, os métodos heurísticos, permitem resolver satisfatoriamente problemas de programação de interesse prático para obtenção de resultados aproximados e confiáveis, apesar de não serem exatos, e consomem menores quantidades de tempo e memória computacionais (Santos e França, 1995). 0 software ArcGIS 10.1 utilizado para execução do modelamento matemático com os dados anteriormente citados, foi capaz de gerar resultados satisfatórios por meio do 
desenvolvimento de uma matriz origemdestino dos custos de caminho mínimo entre todas as facilidades (unidades extrativistas) e os pontos de demandas (comunidades extrativistas) ao longo da rede. Posteriormente, gerou-se um conjunto de soluções aleatórias e aplicou-se uma heurística de substituição de vértices e criação de um grupo de boas soluções.

Quando nenhuma outra melhoria foi possível de ser gerada, a metaheurística retornou a melhor solução encontrada. Desta forma, a combinação de uma matriz editada, uma heurística de substituição de vértices e uma metaheurística de refinamento foi capaz de produzir rapidamente resultados quase ideais (ESRI, 2014).

A base de dados vetoriais utilizada foi composta pelos seguintes temas: comunidades extrativistas de pequi (demandas); localizações potenciais para as instalações das unidades extrativistas (facilidades) e as feições dos sistemas viários (vias pavimentadas e não pavimentadas). Estes dados ao serem modelados permitiram a criação de uma matriz custo levando em consideração o tempo de deslocamento.

As coordenadas das comunidades extrativistas de pequi, foram levantadas de estudo anteriormente desenvolvido na mesma região por Santos et al. (2017) e de informações disponibilizadas pelo Conselho Diretor Pró-Pequi, Núcleo do Pequi, World WideFund for Nature (WWF Brasil), Fundação Pró-Natureza (Funatura), Cooperativa Sertão Veredas (Coopersertão Veredas) e Cooperativa dos Agricultores Familiares e Agroextrativistas do Vale do Peruaçu (Cooperuaçu).

$\begin{array}{rrr}\text { Os locais } & \text { candidatos para } \\ \text { instalação das } & \text { unidades } & \text { de } \\ \text { armazenamento, } & \text { beneficiamento } & \text { e }\end{array}$ distribuição do pequi, foram definidos com adoção de critérios, como regiões próximas de sedes municipais, comunidades extrativistas de pequi, além de demais vilas, aglomerados rurais, povoados e núcleos presentes nas áreas de estudo, com localizações disponibilizadas pelo IBGE (2018).

A fim de delimitar essas regiões foi gerado um "buffer" de 2.000 metros ao redor das localidades mencionadas anteriormente. Esta delimitação visou garantir que as melhores localizações para as unidades de armazenamento, beneficiamento e distribuição do pequi, identificadas por meio do modelamento matemático, possuam estruturas $\mathrm{e}$ recursos mínimos nas proximidades, como eletricidade, água e mão de obra.

As regiões posteriormente foram cruzadas com as áreas potenciais de ocorrências dos pequizeiros na mesorregião norte do estado de Minas Gerais, resultantes de trabalho de pesquisa anteriormente realizada por Magalhães Filho et al. (2017). Uma vez identificados os locais candidatos para instalação das unidades de armazenamento, beneficiamento e distribuição, locais estes representados por polígonos, fez-se necessário converter as referidas áreas em pontos.

Para contornar a situação, as unidades extrativistas candidatas foram definidas como centroides da interseção entre as feições das áreas candidatas para instalação das unidades de armazenamento, beneficiamento e distribuição e uma grade que continha a presença de polígonos de $1.000 \mathrm{x}$ $1.000 \mathrm{~m}$, criada com a ferramenta Fishnet, disponível no ArcToolbox, que permitiu definir as zonas de potenciais em áreas de $1 \mathrm{~km}^{2}$.

As feições dos sistemas viários, constituídos basicamente por vias pavimentadas e não pavimentadas, foram produzidas por meio do traçado das rodovias, disponibilizadas pela plataforma online do DNIT (2013) e de vetorização de demais vias sobre imagens do satélite WorfView II do ano de 2013 disponibilizadas pelo laboratório de geoprocessamento da Universidade Estadual de Montes Claros (Unimontes), polo Montes Claros, Minas Gerais. 
Com a utilização dos dados supracitados, foram desenvolvidos diferentes cenários para as possíveis instalações das unidades para armazenamento, beneficiamento e distribuição do extrativismo do pequi na região norte do estado de Minas Gerais.

As feições vetorizadas dos sistemas viários totalizaram 26.407,32 $\mathrm{km}$, sendo $23.997,43 \mathrm{~km}$ de vias não pavimentadas, $2.408,46 \mathrm{~km}$ de vias pavimentadas e $1,42 \mathrm{~km}$ de travessia de balsa. A fim obter o tempo de deslocamento, foram atribuídos, arbitrariamente, velocidades médias de deslocamentos ao longo de cada tipo de via, sendo $40 \mathrm{~km} / \mathrm{h}$ para as estradas não pavimentas, $80 \mathrm{~km} / \mathrm{h}$ para as estradas pavimentadas e $10 \mathrm{~km} / \mathrm{h}$ para as travessias de balsa. As possíveis localizações para implantação das unidades para coleta, beneficiamento e distribuição do pequi totalizaram 3.711 pontos potenciais.

\section{Resultados e discussão}

Os dados levantados contemplaram 118 comunidades extrativistas e cooperativas de pequi em 22 municípios dos 26 municípios potenciais para a produção do fruto no Norte Estado de Minas Gerais, conforme apresentado na Tabela 2.

Tabela 2. Comunidades e cooperativas extrativistas de pequi investigadas no norte do Estado de Minas Gerais.

\begin{tabular}{|c|c|c|c|}
\hline Nome & Município & Nome & Município \\
\hline Campos & \multirow{31}{*}{ Januária } & Agua Branca & \multirow{11}{*}{ Japonvar } \\
\hline Alegre & & II Barreiros & \\
\hline Vereda Grande II & & Pau Preto & \\
\hline Fabião I & & São bernado I & \\
\hline Vereda Grando I & & Passagem Funda & \\
\hline Olhos D'água & & Cabeceira do Magaí & \\
\hline Araçá & & Rancharia & \\
\hline Pedras & & Ponte do Mangaí & \\
\hline Buritizinho & & Barra da Lagoinha & \\
\hline Lambedouro & & Capivara & \\
\hline Onça & & Genti & \\
\hline Areião/Cooperuaçu & & Passagem Funda II & \multirow{5}{*}{ Japonvar } \\
\hline Vila Lopes & & Porteiras & \\
\hline Cocos & & Santa Rita & \\
\hline Galhos & & Santa Rosa & \\
\hline Jardins & & São Bernardo II & \\
\hline São Joaquim & & Buriti De Santana & \multirow{3}{*}{ Jequitaí } \\
\hline Palmeirinha & & Agua Espraiada & \\
\hline Macaúbas & & Barrocão & \\
\hline Várzea Bonita & & Goiabeira Velha & Lagoa dos Patos \\
\hline Porto Chachueiro & & Lagoinha & Lagoinha \\
\hline Angical & & Novo Horizonte & \multirow{8}{*}{ Lontra } \\
\hline P. A. Imbé & & Palmital & \\
\hline Cabeceira de Mandins & & Santo Antonio & \\
\hline Barra de Mandins & & Tauá & \\
\hline Larga & & Granitinho & \\
\hline P.A. Imbé & & Vila União & \\
\hline Cajueiro & & Sussuapara & \\
\hline Traçadaç & & Pinhão & \\
\hline Pandeiros / COPAAE & & São Bento & \multirow{2}{*}{ Mirabela } \\
\hline ASSUSBAC & & Taboquinha & \\
\hline
\end{tabular}


Tabela 3. Continuação.

\begin{tabular}{|c|c|c|c|}
\hline Nome & Município & Nome & Município \\
\hline COOPERUAÇU & & Muquem & \\
\hline AGRESTE & & Sussuarana & \\
\hline Pedra Preta & \multirow{3}{*}{ Montes Claros } & Coop. Sertão Veredas & \multirow{14}{*}{ Chapada Gaúcha } \\
\hline Lagoinha & & $\begin{array}{l}\text { Comunidade de } \\
\text { Riachinho }\end{array}$ & \\
\hline Coop. Grande Sertão & & Com. de Buraquinhos & \\
\hline Riacho dos Santos & \multirow{8}{*}{ Brasília de Minas } & $\begin{array}{c}\text { Comunidade de } \\
\text { Catarina }\end{array}$ & \\
\hline São Jose & & Comundiade de Pequi & \\
\hline Borá & & Morro do Fogo & \\
\hline Murumbu & & Rio dos Bois & \\
\hline Boa Vista & & Serra das Araras & \\
\hline Vereda da Onça & & Barrocão & \\
\hline Sucuriu & & $\begin{array}{c}\text { Comunidade de } \\
\text { Buracos }\end{array}$ & \\
\hline Sumidozinho & & Cana Brava & \\
\hline Riacho Dos Santos & \multirow{4}{*}{ Campo Azul } & Barro Vermelho & \\
\hline São José & & P.A. São Felix & \\
\hline Sucuriú & & Águas Claras & \\
\hline Sumidorzinho & & Bom Jardim & Ponto Chique \\
\hline Buriti Grande & \multirow{2}{*}{ Francisco Dumont } & ACVS & São Francisco \\
\hline Vila Unida & & Santa Clara & \multirow{2}{*}{ São João da Ponto } \\
\hline Boa Vista & \multirow{2}{*}{ Guaraciama } & Vila dos Carvalhos & \\
\hline Cana Brava & & Raizes & \multirow{2}{*}{ Ubaí } \\
\hline Vereda das Palmeiras & \multirow{2}{*}{ Ibiracatú } & Buritis & \\
\hline Tabuas & & Vereda & \multirow{2}{*}{ Varzelândia } \\
\hline Fazenda Alvorada & \multirow{5}{*}{ Patis } & Bebedouro & \\
\hline Cabeceiro da Cruz & & Vacaria & \multirow{3}{*}{ Coração de Jesus } \\
\hline Buritizinho & & Faveira & \\
\hline Casa nova & & Comunidade do Salto & \\
\hline Pindaiba do Arroz & & Cachoeira do Manteiga & Buritizeiro \\
\hline
\end{tabular}

Após obtenção da base de dados, executou-se uma série de simulações no software ArcGIS 10.1, com variações do número simultâneo das unidades extrativistas instaladas, até o limite de cinco. Foi considerado como fator de impedância o tempo total de deslocamento entre as comunidades extrativistas e as unidades para armazenamento, beneficiamento e distribuição do pequi.

Após as simulações realizadas, foi observada a possibilidade de instalação de cinco unidades (Figura 1) a serem implantadas e os respectivos pontos de oferta (comunidades extrativistas de pequi), onde as linhas roxas representam a alocação das comunidades extrativistas às unidades propostas.

Na primeira simulação realizada, foi observado que o município contemplado para implantação da unidade extrativista foi o município de Japonvar (Figura 1a). Na segunda simulação os municípios de Mirabela e Januária (Figura 1b). Na terceira, os municípios de Chapada Gaúcha, Januária e Mirabela (Figura 1c). Na quarta simulação foi observado que foram contemplados os municípios de Chapada Gaúcha, Januária, Japonvar e Brasília de Minas (Figura 1d). Por fim, na quinta simulação, os municípios Chapada Gaúcha, Japonvar, Brasília de Minas e 
dois pontos no município de Januária (Figura 1e).

No trabalho desenvolvido não foi considerada a capacidade de produção das comunidades extrativistas, haja vista a ausência de dados confiáveis. No entanto, é possível inferir que o aumento do número de unidades para armazenamento, beneficiamento e distribuição do pequi a serem instaladas, promoverá a redução na capacidade de coleta, beneficiamento e distribuição do fruto em cada uma, em contrapartida, a redução do número de unidades proporcionará o aumento da capacidade de absorção da produção em cada uma.

À medida em que se aumenta o número de unidades extrativistas diminui-se o tempo total gasto de deslocamento entre estas $\mathrm{e}$ as comunidades extrativistas, o que consequentemente reduzirá os custos com transporte. Silva et al. (2018), em estudos desenvolvidos com o modelo p-medianas não capacitado na busca de soluções para instalações de ecopontos para coleta de óleo e gordura residual (OGR) em 12 zonas regionais na Cidade de Montes Claros (MG), também observou que os custos totais da solução de cada zona diminuíram à medida que aumentou a quantidade de facilidades encontradas (Tabela 3).

Em modelo desenvolvido por Mapa e Lima (2012), para localização e alocação de fábricas e clientes no estado de São Paulo, o autor também percebeu que os valores de custo total de serviço de transporte diminuíram à medida que se aumentou o número de fábricas, uma vez os clientes ficaram mais próximos, reduzindo os custos de transporte.

Os investimentos iniciais para a instalação de cinco unidades para armazenamento, beneficiamento e distribuição do pequi, com a capacidade de absorção do fruto dividida entre si, poderão ser maiores quando comparado a implantação de uma única unidade com capacidade total de absorção. No entanto, os custos iniciais da primeira alternativa serão compensados ao longo do tempo uma vez que os custos com deslocamento se reduzem em aproximadamente $60 \%$ quando comparado com a implantação de uma única unidade.

Além disso, a implantação de um maior número de unidades provávelmente proporcionará melhor distribuição da oferta de empregos diretos e indiretos e contribuirá para o fortalecimento da economia regional e melhoria da qualidade de vida das comunidades locais.

Dentre os fatores que poderão interferir na decisão quanto ao número de unidades a serem implantadas no norte do Estado de Minas Gerais destacam-se os recursos direcionados pelas políticas públicas à cadeia extrativistas do pequi, bem como das alternativas de operacionalização.

Em relação às localizações para implantação das unidades, recomenda-se que sejam mantidas próximas das coordenadas indicadas. Diante da necessidade de adaptações do modelo heurístico proposto, Pacheco e Cirqueira (2006) recomendam a avaliação do grau de defasagem entre a qualidade da solução do modelo matemático e a solução ótima para o problema in loco.

Arakaki e Lorena (2006) ressaltam que a determinação das melhores localizações para novas facilidades é um importante desafio estratégico para empreendimentos públicos ou privados em função da grande quantidade de capital que deve ser alocada. Esta determinação além de possibilitar a adesão à novos mercados e negócios, proporciona economia de custos, melhora da produtividade e gera impactos positivos tanto para os operadores como para os consumidores, enquanto que localizações ineficientes promovem dificuldades no transporte, mão de obra e gastos adicionais de capital investidos em instalações e operações (Randhawa e West, 1995; Ponboon et al., 2016). 

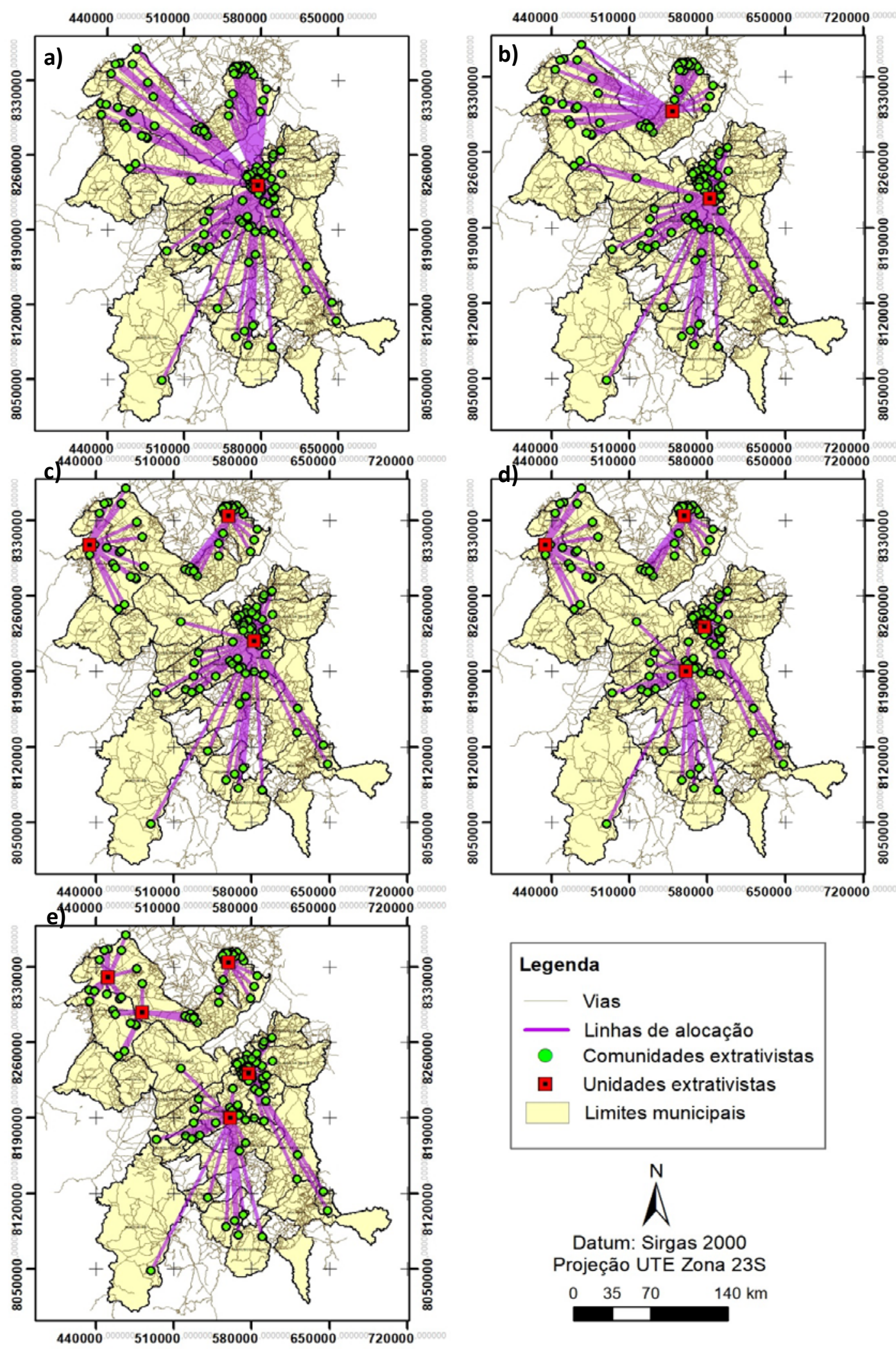

Figura 1. Simulações para instalação de 1 (a), 2 (b), 3 (c), 4 (d) e 5 (e) unidades extrativistas e seus respectivos pontos de oferta. 
Tabela 4. Resultados das simulações com variação do $\mathrm{n}^{0}$ de unidades para armazenamento, beneficiamento e distribuição do pequi alocadas.

\begin{tabular}{|c|c|c|c|c|}
\hline $\begin{array}{c}\text { № de } \\
\text { unidades }\end{array}$ & $\begin{array}{l}\text { Municípios } \\
\text { contemplados }\end{array}$ & Coordenadas & $\begin{array}{c}\text { Tempo } \\
\text { gasto (h) }\end{array}$ & $\begin{array}{c}\text { Número de } \\
\text { comunidades } \\
\text { atendidas } \\
\end{array}$ \\
\hline 1 & Japonvar & $(577311-8231674)$ & 202,67 & 118 \\
\hline \multirow{2}{*}{2} & Mirabela & $(582089-8217422)$ & \multirow{2}{*}{141,94} & 73 \\
\hline & Januária & $(549016-8298120)$ & & 45 \\
\hline \multirow{3}{*}{3} & Chapada Gaúcha & (434584 -8307186) & \multirow{3}{*}{108,09} & 21 \\
\hline & Januária & (558973 -8334031) & & 26 \\
\hline & Mirabela & $(582410-8218086)$ & & 71 \\
\hline \multirow{4}{*}{4} & Chapada Gaúcha & $(434589-8307093)$ & \multirow{4}{*}{92,64} & 21 \\
\hline & Januária & (559134 -8333689) & & 26 \\
\hline & Japonvar & $(577030-8231528)$ & & 44 \\
\hline & Brasília de Minas & $(560838-8190041)$ & & 27 \\
\hline \multirow{5}{*}{5} & Januária 01 & $(559242-8334210)$ & \multirow{5}{*}{82,08} & 20 \\
\hline & Januária 02 & $(481574-8288755)$ & & 15 \\
\hline & Chapada Gaúcha & $(451053-8320482)$ & & 12 \\
\hline & Japonvar & $(577392-8231739)$ & & 44 \\
\hline & Brasília de Minas & $(561017-8189965)$ & & 27 \\
\hline
\end{tabular}

Brasileiro e Chiuffa (2018) destacam que a localização de centros de distribuição é um aspecto relacionado a diversas questões que está direta ou indiretamente associada à produtividade das operações. Os autores mencionam ainda que uma vez definida a rede logística, as futuras operações deverão ocorrer dentro das restrições que a localização das instalações impõe.

Por fim, dentre os objetivos gerais da decisão de localização, além da determinação do número e das melhores localizações para instalações também podem ser identificadas, a capacidade, as responsabilidades de mercado atribuídas a cada instalação e as rotas para o desvio dos fluxos, de acordo com os níveis de custo e/ou capacidade desejados (Amaral et al., 2012; Randhawa e West, 1995). Estas questões são, portanto, oportunidades para o desenvolvimento de novos trabalhos relacionados a cadeia extrativista do pequi no norte de Minas Gerais.

\section{Conflito de interesses}

Os autores declaram não haver conflito de interesses.

\section{Conclusões}

1. A extrema simplicidade da heurística de localização-alocação do tipo p-medianas não capacitada e sua rapidez e eficiência foram confirmadas para a modelagem aplicada;

2. Os testes computacionais realizados demonstraram a efetividade do algoritmo proposto através das ferramentas de apoio à decisão com utilização dos Sistemas de Informações Geográficas;

3. A implantação de cinco unidades de armazenamento, beneficiamento e distribuição do pequi ao longo do norte do estado de Minas Gerais (Januária 1, Januária 2, Japonvar, Brasília de Minas e Jequitaí) é a simulação mais vantajosa, uma vez que o somatório do tempo de deslocamento ao longo do sistema logístico foi o menor dentre todas as outras simulações, o que 
acarretará menores custos com deslocamento.

4. Pode ser implantado um menor número de unidades de armazenamento, beneficiamento e distribuição do pequi para garantir diminuição do custo inicial de investimento. No entanto, os custos de deslocamento provavelmente serão inversamente proporcionais ao número de unidades implantadas.

5. Para a definição do número de unidades a serem implantadas e dos investimentos a serem realizados, devem ser considerados diferentes fatores, tais como a disponibilidade de recursos e a capacidade de fortalecimento da estrutura de mercado com a implantação das unidades.

\section{Conflitos de interesses}

Os autores declaram não haver conflitos de interesses.

\section{Referências}

Afonso, S. R.; Ângelo, H. Mercado dos produtos florestais não-madereiros do cerrado brasileiro. Ciências Florestais, v. 19, n. 3, p. 315-326, 2009. https://doi.org/ 10.5902/19805098887

Amaral, M.; Almeida, M. S.; Morabito, R. Um modelo de fluxos e localização de terminais intermodais para escoamento da soja brasileira destinada à exportação. Gestão \& Produção, v. 19, n. 2, p. 717-732, 2012. https://doi.org/10.1590/S0104-530X2012 000400005

Arakaki, R. G. I.; Lorena, L. A. N. Uma heurística de localização-localização (HLA) para problemas de localização de facilidades. Produção, v. 16, n. 2, p. 319-328, 2006. https://doi.org/10.1590/S0103-65132006 000200011

Brasileiro, L. A.; Chiuffa, V. P. D. Simulation of the optimal location for a distribution center of e-commerce operations in São Paulo State. IEEE Latin America Transactions, v. 16, n. 6, 2018. https://doi.org/10.1109/TLA. 2018.8444401
Brondani, A. E.; França, F. A. M.; Netto, P. O. B.; J., S.; Kopp Junior, R. V. Alocação de unidades urbanas de lazer por um modelo p-medianas. Revista Eletrônica Pesquisa Operacional para o Desenvolvimento, v. 5, n. 2, p. 209-223, 2013.

Calache, L. D. D. R.; Camargo, V. C. B. Proposta de um método de localização e alocação de pontos de coleta seletiva. Revista Eletrônica Pesquisa Operacional para o Desenvolvimento, v. 8, n. 2, p. 137-162, 2016.

Camara, M. V. O.; Ribeiro, G. M.; Quadros, S. G. R.; Hora, C. A. H.; Nascimento, C. J. F.; Abramides, C. A. Modelagem matemática aplicada no planejamento de pesquisa de tráfego rodoviário. Revista Produção Online, v. 16, n. 2, p. 722-742, 2016.

Candido, P. A.; Malafaia, G. C.; Rezende, M. L. A exploração do pequi na região norte de Minas Gerais: abordagem por meio do Sistema Agroalimentar Localizado. Revista Ideas, v. 5, n. 2, p. 118-138, 2012.

Costa, B. B.; Nassi, C. D.; Ribeiro, G. M. A methodology for location of logistics platforms using Geographic Information Systems. Journal of Traffic and Logistics Engineering, v. 1, n. 2, p. 104-110, 2013.

Crainic, T.; Laporte, G. Planning models for freight transportation. European Journal of Operational Research, v. 97, n. 3, p. 409438, 1997. https://doi.org/10.1016/S03772217(96)00298-6

DNIT - Departamento Nacional de Infraestrutura de Transportes. ShapefilesBrasil, 2013. Disponível em: <http://www.dnit.gov.br/mapas-multimo dais/shapefiles>. Acesso em: 10 fev. 2019.

Espírito Santo, M. M.; Leite, M. E.; Silva, J. O.; Barbosa, R. S.; R2ocha, A. M.; Anaya, F. C. Understanding patterns of land-cover change in the Brazilian Cerrado from 2000 to 2015. Philosophical Transactions of the Royal Society B, v. 371, $\quad$ n. 1703, 2016. https://doi.org/10.1098/rstb.2015.0435

ESRI - Environmental Systems Research Institute. Algoritmos usados pela extensão do ArcGIS Network Analyst, 2014. Disponível em: <http://desktop.arcgis.com/en/arcmap/ latest/extensions/network-analyst/ algorithms-used-by-network-analyst.htm>. Acesso em: 10 fev. 2019. 
Garey, M. R.; Jonhson, D. S. Computers and intractability: A guide to the theory of NPcompleteness. San Francisco, CA: W. H. Freeman and Co., 1979.

IBGE - Instituto Brasileiro de Geografia e Estatística. Estado de Minas Gerais - Meso e microrregiões do IBGE, 2019. Disponível em: <http://mg.gov.br/sites/default/files/ paginas/arquivos/2016/ligminas_10_2_04_li stamesomicro.pdf>. Acesso em: 17 jan. 2019.

IBGE - Instituto Brasileiro de Geografia e Estatística. Produção da Extração Vegetal e da Silvicultura - PEVS, 2019. Disponível em: $<$ https://www.ibge.gov.br/estatisticasnovop ortal/economicas/agricultura-e-pecuaria/ 9105-producao-da-extracao-vegetal-edasilvicultura.html>. Acesso em: 17 jan. 2018.

Magalhães Filho, R.; Borges, M. G.; Leite, M. R. Ocorrência do pequizeiro (Caryocar brasiliense) no Norte de Minas Gerais. Revista Desenvolvimento Social, v. 21, n. 2, p. 117-125, 2017.

Mapa, S. M. S.; Lima, R. S. Uso combinado de sistemas de informações geográficas para transportes e programação linear inteira mista em problemas de localização de instalações. Gestão \& Produção, v. 19, n. 1, p. 119-136, 2012. https://doi.org/ 10.1590/S0104-530X2012000100009

Pacheco, R. F.; Cirqueira, L. Z. Solução simultânea de problemas logísticos de localização de depósitos e centralização de estoques. Produção, v. 16, n. 3, p. 481-492, 2006. https://doi.org/10.1590/S0103-6513 2006000300010

Pedrozo, E. A.; Silva, T. N.; Sato, S. A. S.; Oliveira, N. D. A. Produtos florestais não madeiráveis (PFNMS): as filières do açaí e da castanha da Amazônia. Revista de Administração e Negócios da Amazônia, v. 3, n. 2, p. 88-112, 2011.

Ponboon, A.; Qureshi, A. G., Taniguchi, E. Evaluation of cost structure and impact of parameters in location routing problem with time Windows. Transportation Research
Procedia, v. 12, p. 213-226, 2016. https://doi.org/10.1016/j.trpro.2016.02.060

Randhawa, S. U.; West, T. M. An integrated approach to facility location problems. Computers \& Industrial Engineering, v. 29, n. $1 / 4$, p. 261-265, 1995. https://doi.org/ 10.1016/0360-8352(95)00082-C

Reboita, M. S.; Rodrigues. M.; Silva. L. F.; Alves. M. A. Aspectos climáticos do Estado de Minas Gerais. Revista Brasileira de

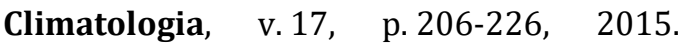
https://doi.org/10.5380/abclima.v17i0.41493

Rodrigues, L. J.; Paula, N. R. F.; Pinto, D. M.; Vilas Boas, E. V. B. Growth and maturation of pequi fruit of the Brazilian cerrado. Food Science and Technology, v. 35, n. 1, p. 1117, 2015. https://doi.org/10.1590/1678457X.6378

Santos, H. C. M.; Franca, P. M. Meta-heurística para programação da produção com tempos de preparação dependentes da sequência. Gestão \& Produção, v. 2, n. 3, p. 228-243, 1995. https://doi.org/10.1590/S0104530X1995000300001

Santos, M. T. P.; Fonseca, G. L.; Almeida, M. I. S. Dinâmica de exploração do pequi nos municípios produtores na Mesorregião Norte de Minas. Anais do V Colóquio Cidade e Região Sociedade e Ambiente: Dinâmicas Urbanas e Rurais, Montes Claros, 2017.

Silva, J. V. L.; Santos Neto, N. F. S.; Pereira, A. J. Utilização do Sistema de Informação Geográfica e o modelo p-medianas não capacitado na busca de soluções para instalações de ecopontos para coleta de OGR. Revista Mundi - Engenharia, Tecnologia e Gestão, v. 3, n. 2, p. 74-1-74-14, 2018. https://doi.org/10.21575/25254782rmetg2 018vol3n2540

Silva, M. N. S.; Tubaldini, M. A. S. O ouro do Cerrado: a dinâmica do extrativismo do pequi no Norte de Minas Gerais. Revista Geoaraguaia, v. 3, p. 293-317, p. 293-317, 2013. 